\title{
CME-Fragen bei CMIE.thiemede
}

Viel Erfolg bei Ihrer CME-Teilnahme unter http://cme.thieme.de

Bitte informieren Sie sich über die genaue Gültigkeitsdauer unter http://cme.thieme.de

Sollten Sie Fragen zur Online-Teilnahme haben, unter http://cme.thieme.de/hilfe

finden Sie eine ausführliche Anleitung.

VNR 2760512017152370484

\section{Frage 1}

Welche Darmkrebs-Screening-Methoden sind Kassenleistung?
A FOBT
B Koloskopie
C Kolonkapsel
D FOBT und Koloskopie
E CT-Kolonografie

\section{Frage 2}

Welche Antwort trifft zu? Das Darmkrebsrisiko ist besonders abhängig von
A Ernährungsgewohnheiten
B Familiärer Belastung
C Hohem Alter
D Geschlecht
E Nikotinkonsum

\section{Frage 3}

Welche Antwort zur Darmkrebsvorsorge ist falsch?

A Die Krankenkassen in Deutschland übernehmen ab dem 50. Lebensjahr einen Test auf nicht sichtbares Blut im Stuhl.

B Die Stuhluntersuchung kann jährlich bis zum 55. Lebensjahr wiederholt werden.

C Mit 55 Jahren besteht der Anspruch auf eine Vorsorgedarmspiegelung.

D Menschen mit familiärer Darmkrebsbelastung sollten bereits mit 40-45 Jahren eine Darmspiegelung erhalten.

E Menschen mit familiärer Darmkrebsbelastung sollten primär eine Stuhluntersuchung erhalten.

\section{Frage 4}

Welche Antwort ist richtig? Die Erfassung des 10-Jahres-Risikos einer Person für tödliche kardiovaskuläre Erkrankungen mit dem SCORE-Modell berücksichtigt ...
A Alter, systolischen Blutdruck, Geschlecht, Raucher/Nicht- raucher und Triglyceride.
B Alter, systolischen Blutdruck, Geschlecht, Raucher/Nicht- raucher und LDL-Cholesterin.
C Alter, systolischen Blutdruck, Geschlecht, Raucher/Nicht- raucher und Gesamt-Cholesterin.

D Alter, systolischen und diastolischen Blutdruck, Raucher/ Nichtraucher, Geschlecht und Gesamt-Cholesterin.

E Alter, systolischen Blutdruck, Raucher/Nichtraucher und Geschlecht.

\section{Frage 5}

Welche Antwort ist richtig? Das Screening nach einem primären Hyperaldosteronismus mittels Bestimmung des AldosteronRenin-Quotienten erfolgt ...:
A unter laufender Therapie mit einem Mineralokortikoid-Rezep- tor-Antagonisten.
B nach Pausieren einer Therapie mit einem Schleifendiuretikum oder Betablocker für mindestens 4 Tage.
C unter vorhergehender Reduktion der Kochsalzzufuhr.
D bevorzugt unter einer Therapie mit einem Renin-Blocker.
E möglichst nach Ausgleich einer Hypokaliämie.

\section{Frage 6}

Welche Antwort ist falsch? Labordiagnostik bei arterieller Hypertonie erfasst u.a. folgende kardiovaskuläre Risikofaktoren
A Nüchternblutglukose
B $\mathrm{HbA}_{1 \mathrm{c}}$
C HIV-Infektion
D Lipidstatus
E Chronische Nierenerkrankung

\section{Frage 7}

Welche Antwort ist falsch? Labordiagnostik bei arterieller Hypertonie erfasst im Screening nach sekundären Hypertonieformen u. a.
A Renale Hypertonieformen
B Cushing Syndrom
C Primärer Hyperparathyreoidismus
D Helicobacter pylori
E Schilddrüsenfunktionsstörungen 


\section{CME-Fragen bei CME.thieme.de}

\section{Fortsetzung ...}

VNR 2760512017152370484

\section{Frage 8}

Messung der Nierenfunktion: Welche Antwort zu exogenen Filtrationsmarkern ist falsch?
A Inulin ist der Goldstandard unter den Goldstandards
B lothalamat ist günstig und auch ohne Urinclearance möglich
C Iohexol gilt als genauer als lothalamatclearance
D Cr-EDTA ist in Europa nicht verfügbar
E DTPA ist in den USA breit verfügbar

\section{Frage 9}

Welche Aussage zur Schätzung der Nierenfunktion (eGFR) trifft nicht zu?

A Im klinischen Alltag wird die GFR in Deutschland meist mittels mathematischer Formeln geschätzt.

B Schätzformeln können nur für die Personengruppe gelten, in der sie entwickelt wurde, d.h. eine Schätzformel, die ausschließlich in Kranken entwickelt wurde, unterschätzt die GFR bei Gesunden.

C Die Kombination von Kreatinin und Cystatin C führt in bestimmten Populationen (z. B. alte Menschen) zu einer präziseren GFR-Bestimmung als ein Marker allein.

D Für die vielversprechenden „Full Age Spectrum“ (FAS)-Gleichungen, die für das gesamte Altersspektrum von 2-100 Jahren angewendet werden können, fehlen derzeit noch externe Validierungen in unterschiedlichen Populationen.

E Auf der Suche nach dem optimalen endogenen Biomarker haben das Beta-Trace-Protein (BTP) sowie Beta-2-Mikroglobulin (B2M) hervorragende Ergebnisse erzielt und werden in Kürze zugelassen.

\section{Frage 10}

Welche Antwort zu endogenen Biomarkern trifft nicht zu?
A Für Kinder sind CysC und Crea/CysC geeignete Verfahren.
B Für Erwachsene sind Kreatinin, CysC und Crea/CysC geeignete Verfahren.
C Für alte Menschen (70+) ist Crea/CysC ein geeignetes Verfahren.
D Für Leistungssportler sind Kreatinin und mGFR geeignete Verfahren.
E Unter Chemotherapie sind Kreatinin und mGFR geeignete Verfahren. 